\title{
网构软件技术体系：一种以体系结构为中 心的途径
}

杨芙清 ${ }^{(1) *}$ ，吕建 ${ }^{(3)}$ ，梅宏 ${ }^{(1)}$

(1) 北京大学高可信软件技术教育部重点实验室, 北京 100871;

(2) 北京大学信息科学技术学院软件研究所, 北京 100871 ;

(3) 南京大学计算机软件新技术国家重点实验室, 南京 210093;

(4) 南京大学计算机软件研究所, 南京 210093

*E-mail: yang@pku.edu.cn

收稿日期: 2007-12-24; 接受日期: 2008-03-06

国家重点基础研究发展规划资助项目(批准号: 2002CB312000)

摘要 作为 Internet 环境下的一种新型的软件形态，网构软件对传统的软 件开发理论、方法与技术提出了一系列的挑战. 我国学者在国家“973”计划支 持下, 经过 5 年的研究与实践, 形成了一套以体系结构为中心的网构软件技 术体系。在网构软件模型方面, 通过开放协同模型、情境驱动模型和智能可 信模型实现了网构软件基本实体的构件化、主体化、服务化以及实体间开放 的结构化的按需协同; 在网构软件中间件方面，以构件化的中间件平台体系 为基础, 实现了支持网构软件基本实体运行的容器、支持按需协同的运行时 体系结构、以及基于反射的网构软件自治管理; 在网构软件开发方法方面, 以全生命周期软件体系结构为核心，通过模型驱动的方式支持网构软件基本 实体和结构化协同的高效开发、以及遗产系统向网构软件的半自动转换, 并 通过领域建模实现了网构软件无序资源的有序组织.

关键词

网构软件

构件

软件体系结构

Agent

软件中间件

软件是客观事物的一种虚拟反映, 是知识的固化、凝炼和体现. 软件的这种本质, 驱动着 软件基本模型不断追求更具表达能力, 更符合人类思维模式, 更具可构造性和演化性的软件 结构, 如, 结构化软件试图适应人分析问题和解决问题的思维模式, 面向对象软件试图适应客 观世界的问题结构. 另一方面, 软件的主要作用是指导计算机系统进行问题求解并实施相应 的管理, 这就驱动着软件不断追求更高效合理地发挥硬件资源所提供的计算能力. 以操作系 统为例, 首先, 随着计算机硬件的发展, 单机操作系统从最早的引导程序到管理计算机资源的 
管理程序, 再发展到发挥 CPU 能力及外设功用的多道程序支撑, 最后发展为追求计算机软硬 件高效利用的资源管理系统. 网络操作系统的出现主要是用以支持多个计算机之间的网络通 信, 实现资源共享, 而并行操作系统是为了管理大规模并行处理系统. 同时, 无线通信、信息 家电的发展在一定程度上又促进了嵌入式操作系统的发展. 简言之, 随着应用领域和信息技 术的持续发展变化, 软件技术及系统也不断地升级、演化, 呈现出不同的形态.

进入 21 世纪, 以Internet为代表的网络逐渐融入人类社会的方方面面, 极大地促进了全球 化的广度和深度, 为信息技术与应用扩展了发展空间. 另一方面, Internet正在成长为一台由数 量巨大且日益增多的计算设备所组成的 “统一的计算机”, 与传统计算机系统相比, Internet为应 用领域问题求解所能提供的支持在量与质上均有飞跃. 为了适应这些应用领域及信息技术方 面的重大变革, 软件系统开始呈现出一种柔性可演化、连续反应式、多目标自适应的新系统形 态. 从技术的角度看, 在面向对象、软件构件等技术支持下的软件实体以主体化的软件服务形 式存在于Internet的各个节点之上, 各个软件实体相互间通过协同机制进行跨网络的互连、互 通、协作和联盟, 从而形成一种与WWW相类似的软件Web(software Web). 我们将这样一种 Internet环境下的新的软件形态称为网构软件(Internetware) ${ }^{[1,2]}$.

具体而言, 网构软件包括一组分布于 Internet 环境下各个节点的、具有主体化特征的软件 实体, 以及一组用于支撑这些软件实体以各种交互方式进行协同的连接子. 这些实体能够感知 外部环境的变化, 通过体系结构演化的方法(主要包括软件实体与连接子的增加、减少与演化、 以及系统拓扑结构的变化等)来适应外部环境的变化, 展示上下文适应的行为, 从而使系统能够 以足够满意度来满足用户的多样性目标. 网构软件这种与传统软件迥异的形态, 在微观上表现 为实体之间按需协同的行为模式，在宏观上表现为实体自发形成应用领域的组织模式. 相应地， 网构软件的开发活动呈现为通过将原本 “无序”的基础软件资源组合为 “有序”的基本系统, 随着 时间推移, 这些系统和资源在功能、质量、数量上的变化导致它们再次呈现出 “无序”的状态, 这 种由“无序”到“有序”的过程往复循环，基本上是一种自底向上、由内向外的螺旋方式.

从网构软件的角度来考察当今有代表性的软件工程方法学, 可以发现传统的软件工程方 法学体系由于其本质上是一种静态和封闭的框架体系, 难以适应 Internet 开放、动态、难控以 及应用领域全球化、个性化、持续成长等特点. 本质上, 网构软件是传统软件在 Internet 环境 下的一种自然延伸, 其基本特征、概念框架、逻辑内涵、关键技术可视为未来软件方法与技术 的一种抽象规约. 在国家重点基础研究发展规划(973)的支持下, 来自北京大学、南京大学、清 华大学、中国科学院软件研究所、中国科学院数学研究所、华东师范大学、东南大学、大连 理工大学、上海交通大学等单位的研究人员以我国软件产业需支持信息化建设和现代服务业 为主要应用目标, 提出了 “Internet 环境下基于 Agent 的软件中间件理论和方法研究”, 并形成了 一套以体系结构为中心的网构软件技术体系, 主要包括 3 个方面的成果: 在网构软件实体模型 方面, 剥离对开放环境以及其他实体的固化假设, 以解除实体之间以及实体与环境之间的紧 密耦合, 进而引入自主决策机制来增强实体的主体化特性; 在网构软件实体协同方面, 针对面 向对象方法调用受体固定、过程同步、实现单一等缺点, 对其在开放网络环境下予以按需重新 解释, 即, 采用基于软件体系结构的显式的协同程序设计, 为软件实体之间灵活、松耦合的交 
互提供可能; 在网构软件运行平台(中间件)方面, 通过容器和运行时软件体系结构分别具体化 网构软件基本实体和按需协同, 并通过构件化平台、全反射框架、自治回路等关键技术实现网 构软件系统化的自治管理; 在网构软件开发方法方面, 提出了全生命周期软件体系结构以适 应网构软件开发重心从软件交付前转移到交付后的重大变化, 通过以体系结构为中心的组装 方法支持网构软件基本实体和按需协同的开发, 采用领域建模技术对无序的网构软件实体进 行有序组织.

\section{1 网构软件模型}

软件模型是软件方法学的核心, 集中体现了一种软件方法及其技术支撑体系以何种基本 视角来看待软件系统之成分构成与运行机理. 作为开放、动态和难控网络环境下的分布式软件 系统的一种抽象, 网构软件在构成、运行、正确性、开发、可信性、生存周期等方面与经典软 件系统相比均存在差异:

- 在系统构成方面, 网构软件是开放的和反射的(reflective). 开放性指网构软件由分布在 广域范围内的、通常由第三方提供的、具有主体化服务特征的一组软件实体, 通过各类风格各 异的协同连接子加以互连互通而形成的联盟; 反射性是指网构软件包括两个层次的内容, 即目 标层与元层(或曰控制层). 目标层不仅包括经典软件系统的范围, 而且还包括显式的环境抽象. 元层则主要包括用于反映环境变化的感知机制部分和用于调节系统行为的演化机制部分.

- 在系统运行方面, 网构软件能够主动适应情境. 目标层系统的运行, 主要用于满足某一 时段内用户的需求; 元层根据环境的变化对运行系统按照所需的指标加以调整与演化, 从而使 目标层系统在下一个时段能更好地满足用户的需求. 如此不断往复, 系统的运行过程也是系统 不断演进的过程, 这也导致在生存周期方面, 网构软件强调一种从演化为主的动态模型.

口 在系统正确性方面, 通过不断的系统演化, 网构软件所追求的刚性的正确性要求逐步 转向随时段变化而变化的用户柔性满意度.

- 在系统开发方面, 软件开发过程就是不断获取最佳的服务与增值服务的过程, 在此过 程中, 由主体化的软件实体与分离的协同机制所支持的成长式开发方式本质上蕴含了基于构 件组装的软件复用思想.

- 在系统可信性方面, 在经典的安全与可靠保障技术基础上, 强调一种在开放环境下基 于历史运行信息评价的柔性可信度量、推断与应用机制.

考察经典、主流的面向对象软件模型, 由于其产生于静态、封闭、可控的环境, 从而具有 有限自主、固定封装、交互单调、结构紧耦、离线应变等特征. 虽然近年来在分布对象技术、 面向侧面(aspect)编程等方面取得了一些进展, 但其根本特征没有改变, 无法直接支持网构软 件. 为此, 我们基于面向对象模型, 提出了一种基于Agent、以软件体系结构为中心的网构软件 模型(技术细节请参考文献[3 9], 如图 1 所示.

\section{1 基本构件模型}

网构软件基本实体首先是一个构件, 以确保单个实体可以独立部署、运行与演化. 与传统 软件构件类似, 网构软件基本实体也具有业务接口, 以提供计算、控制或连接等功能. 与传统 
软件构件不同的是, 网构软件基本实体具有反射接口, 以提供监测、分析、规划、调整其业务 功能的反射功能(也称为元计算), 进而实现网构软件的自主性、演化性、协同性、多态性和反 应性等特性. Internet “天然”的异构性决定了网构软件基本实体所具备的特性不尽相同，因此， 网构软件构件模型自身是可定制和可扩展的, 如, 仅提供业务功能而不具备任何反射与推理 能力的软件实体, 可以认为是网构软件实体的一种基础形态; 定义了构件元模型且允许通过 反射接口访问的软件实体则具备个体感知能力; 理解环境元模型且可通过反射接口访问的软 件实体具备环境感知能力; 可调整自身状态或行为的软件实体具备个体适应能力等等. 这些 网构软件特性的原理与实现则由开放协同模型、情境驱动模型和智能可信模型来规范.

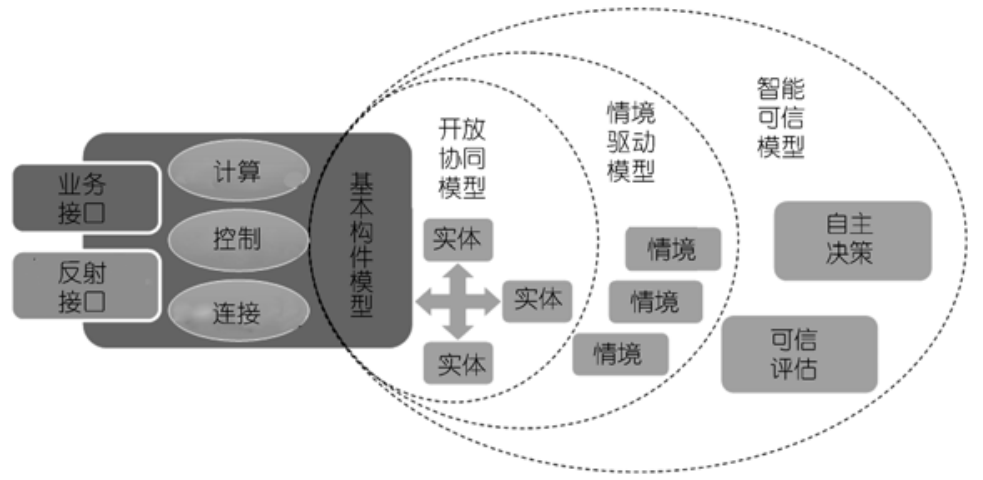

图1 网构软件模型

\section{2 开放协同模型}

开放协同软件模型的核心就是给面向对象程序模型中的核心概念“(对象.消息)结构”赋予 开放环境下的新含义, 以此来适应开放网络环境的需要. 它具有如下特征：在结构上, 软件实 体与协同部分在构成上是分离的, 协同逻辑基于软件体系结构成为显式的独立实体; 在开发 上, 软件实体与协同部分可由服务提供者和服务集成者分别开发, 面向服务提供者的软件实 体编程可由成熟的面向对象技术支撑, 由现有的标准技术, 如 Web Services 等, 加以封装, 而 面向服务集成者的协同程序设计将由内置式软件体系结构、多模式共存的灵活交互方式和面 向协同的服务剪裁与增强机制来支持; 在应变上, 实体部分主要通过大量新生的、可供选择的 优质服务来不断完善或淘汰旧的服务, 而协同部分则通过对软件服务的灵活剪裁、多样性的综 合集成、以及分层次的协同演化来适应外部环境的变化.

\section{3 情境驱动模型}

情境驱动模型的研究目标是在开放协同模型的基础上, 分析外部环境的特征及其多样的 变化模式, 建立环境模型的框架结构, 在此基础上, 以反应式服务计算为突破点, 建立开放协 同模型与环境模型的交互计算模式和自适应演化模式, 从而形成相应的结构、方法与技术. 情 境驱动系统的构造需要通过对网构软件系统与运行环境及使用者之间不断演进的互动模式进 行感知和分析, 归纳出情境驱动的行为模式, 进而利用上述框架和自适应体系结构技术实现 
情境感知和主动适应. 在实现上, 通过基于 Agent 的情境信息收集和处理并结合智能化的体系 结构演化驱动来达到上述目标.

\section{4 智能可信模型}

智能可信计算模型就是在情境驱动模型的基础上, 将可信计算框架和智能技术等引入, 解决开放环境下软件的可信性、个性化、自发成长等方面的问题. 也就是在经典的具有“关注 单一属性、开发者主导、以刚性规约为主”等特征的保障机制与支撑手段的基础上，发展一种 具有综合考虑多种需求、基于实证、评价标准灵活等特征的、适合于网构软件系统的新型保 障机制与支撑手段. 技术上需要包括网构软件实体与系统的信任描述框架和度量模型, 基于 Agent 的信任管理，可信的系统(半)自动组合与演化等机制的支撑.

\section{2 网构软件中间件}

软件中间件(简称为中间件)是指网络环境下处于操作系统等系统软件和应用软件之间的 一种起连接作用的分布式软件, 主要解决异构网络环境下分布式应用软件的互连与互操作问 题, 它可屏蔽实现细节, 提高应用系统的易移植性. 随着 Internet 的快速发展与广泛应用, 中间 件技术、产品、应用、标准逐步繁荣, 其定义正在走出狭义空间, 逐步形成更为广义的内涵, 成 为一种 Internet 环境下的新型系统软件, 且呈现出如下发展趋势:

- 平台化: 越来越多地向传统运行层(操作系统)渗透, 提供更强的运行支撑, 形成屏蔽 操作系统、网络协议、数据库管理系统的网络运行平台;

- 泛在化: 中间件从传统的企业计算延伸到对等计算、普适计算、网格计算、服务计算 等新型计算模型，使得中间件广泛存在于各种计算模型与系统中;

- 领域化: 不再局限于提供适用于大多数应用的支持机制, 那些适用于某个领域内大部 分应用的支持机制(这些机制往往无法在其他领域使用)也开始得到重视;

- 模型化: 中间件对于整个软件生命周期的影响得到重视, 开始考虑将中间件抽象成不 同的模型以支持高层设计、测试、应用部署甚至需求分析等开发活动.

中间件的繁荣及其发展趋势决定了其在网构软件技术体系中的地位和作用：作为 “操作系 统”负责网构软件系统的运行与管理. 具体而言, 网构软件中间件应具备两种主要能力: 其一, 网构软件模型的实现, 即, 如何将前面建立的网构软件基本实体变为可运行的实体, 以及如何 在软件体系结构的管控下实施这些运行实体之间的按需协同; 其二, 网构软件系统的管理, 即, 在开放、动态、难控的Internet环境中如何保证网构软件系统的运行能够满足预期的功能性、 性能、可靠性、安全性等可信指标. 为此, 我们建立了如图 2 所示的网构软件中间件模型(技术 细节请参考文献[10 18]):

\section{1 网构软件基本实体的实现模型}

容器是网构软件基本实体运行时所处的空间, 负责实体生命周期的管理(如类装载、实例 化、缓存、释放等)、实体运行上下文的管理(如访问引用上下文、数据库连接等)、以及实体 的基本业务与高级策略之间的绑定(与互操作协议绑定以允许通过 RMI, HTTP, SOAP 等访问 
业务接口，与约束服务绑定以实现访问控制、数据持久化、事务等约束，与智能设施绑定以实 现业务规则自动调整, 与可信机制绑定以实现信誉评估等). 值得指出的是, 基本业务的实现 体、高级策略的实现体、以及各个绑定均可在线调整, 以体现网构软件的多变性.

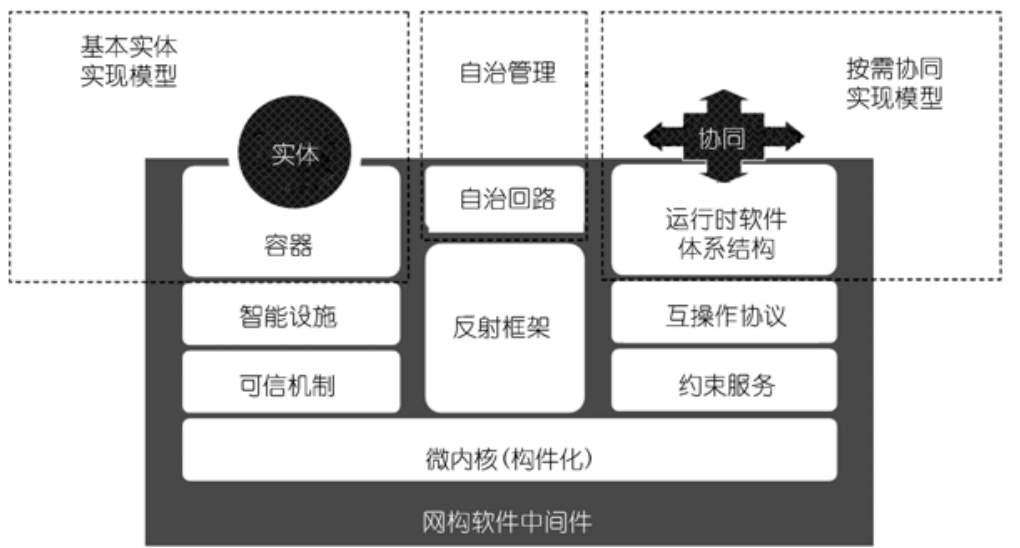

图2 网构软件中间件模型

\section{2 网构软件按需协同的实现模型}

运行时软件体系结构 RSA(runtime software architecture) 是中间件提供的一种运行实体, 由 于中间件对整个系统的深度控制而与运行系统之间建立了一种因果关联关系, 这种关系确保 RSA 的变化会实时转换为运行系统的变化, 反之亦然. 每个网构软件基本实体均抽象并实例 化为一个 RSA 构件, 两个实体之间的交互则抽象并实例化为一个 RSA 连接子, 从而将网构软 件实体之间的按需协同实现为 RSA. 宏观上, 所有网构软件实体之间的协同形成了一个 Internet 规模的 RSA. 微观上, 既可调整 RSA 的局部以显式驱动实体之间的协同, 也允许实体 自主协同(但该协同由于实现为 RSA 的一部分而受到管控).

因果关联关系的建立有反射式编程模型和反射式中间件两种主要途径, 各有利弊. 我们 以反射式中间件为主, 整合了反射式编程模型, 实现了一种全反射框架以提高因果关联关系 的广度和深度, 从而满足网构软件按需协同的需要.

\section{3 网构软件中间件的自治管理}

中间件的繁荣以及网构软件丰富的特性对中间件的管理提出了两方面的挑战. 一方面, 网构软件中间件自身能力多样化、复杂化, 定制和扩展中间件能力并确保这些能力的可信变得 日益复杂、难控. 另一方面, 中间件在整个系统所占比重日益增大, 中间件管理的好坏直接决 定了整个系统可信指标的好坏, 因此, 从整个系统的角度管理中间件成为必需. 而管理目标的 弹性化以及运行环境的多变性进一步提高了中间件管理的复杂度, 这就要求中间件具备自治 管理的能力, 即, 尽可能自动实施管理手段以减少甚至避免人的参与.

针对中间件自治管理的可管理性、可操作性和可信性 3 个基本性质, 我们建立了以体系结 构为中心的中间件自治回路, 即, 建模中间件及其应用的软件体系结构以定义中间件的管理 
边界及内涵, 基于运行时软件体系结构监测和调整运行系统, 评估软件体系结构以确保自治 的正确性和效用性. 对于自治计算最关键的分析和决策, 我们首先通过软件体系结构及决策 的规范化描述将隐性、劣构的知识转变为显性、良构的知识, 其中, 常识性的、领域特定的、 应用特定的知识一般分别描述为体系结构风格、领域特定的体系结构、应用体系结构. 具体的 调整方案既可采用动态软件体系结构这种命令式描述, 也可采用自适应软件体系结构这种说 明式描述, 即, 由给定知识自动推导出调整方案, 如, 由〈事件, 条件, 动作〉规则推导出给定 事件和条件下应执行的动作, 由不良模式和良好模式推导出体系结构局部重构的方案, 由两 种给定风格推导出风格融合的调整方案等.

\section{4 网构软件中间件平台构件化}

不难发现, 上述能力均要求网构软件中间件平台具有清晰优良的结构, 为此, 我们建立了 基于微内核的中间件构件化平台体系. 容器、协议、服务、设施、机制、框架等中间件能力的 具体实现均封装为平台构件, 为了支持这些平台构件的按需定制、扩展以及自治管理, 我们设 计了一个微内核, 包括注册/注销接口以允许平台服务动态插入、命名接口和查询接口以定位 给定的平台构件、一种总线机制以允许通过特定的元编程接口传递对平台构件的调用请求、 平台构件生命周期管理机制、元数据接口和元编程机制等. 值得指出的是, 和操作系统中微内 核的作用不同，网构软件中间件微内核不提供内存管理、进程间通信、I/O 及中断管理等功能, 而仅提供平台构件的封装、集成和协调等基本管理机制.

\section{3 网构软件开发方法}

Internet开放、动态、难控的特性以及用户使用方式的个性化要求, 决定了网构软件的开 发不同于传统软件开发的“一次成型”式, 具体表现为, 网构软件在发布之后, 能够感知外部网 络环境的动态变化, 并随着这种变化按照功能指标、性能指标和可信性指标等进行静态的调整 和动态的演化, 以使系统具有尽可能高的用户满意度, 而且, 由于用户需求的多样化和个性化 以及投资回收等因素, 一个软件系统往往存在时间较长, 因此, 网构软件的演化过程也在长时 间内持续不断. 显然, 开发具有上述技术特点的网构软件面临着开发过程、开发方法以及支持 技术 3 个方面的挑战. 为此, 我们建立了如图 3 所示的网构软件开发方法体系(技术细节请参考 文献[19 28]).

\section{1 全生命周期软件体系结构}

在传统的软件工程体系中, 软件生命周期概念所强调的是从问题提出到软件交付的整个 开发过程的重要性, 而对于交付之后的软件变化过程往往只采用“软件维护”加以简单概括. 这样一种软件生命周期概念对处于静态封闭环境下的软件系统的开发是合适的, 但对处于 Internet 开放、动态和多变环境下网构软件系统的开发则有明显的局限性. 首先, 新的软件实 体往往通过已有的软件实体组装而成, 由于这些实体彼此独立、自主运行, 不受任何机构或组 织的统一控制, 因此, 在系统交付运行之前, 很难由这些被组装的软件实体准确无误地推演出 目标系统的功能和质量. 其二, 环境的开放、动态和多变决定了软件实体及其之间的协同均面 临着诸多变化, 不论这些变化能否在运行前准确预知, 运行系统都不得不进行持续的适应性 


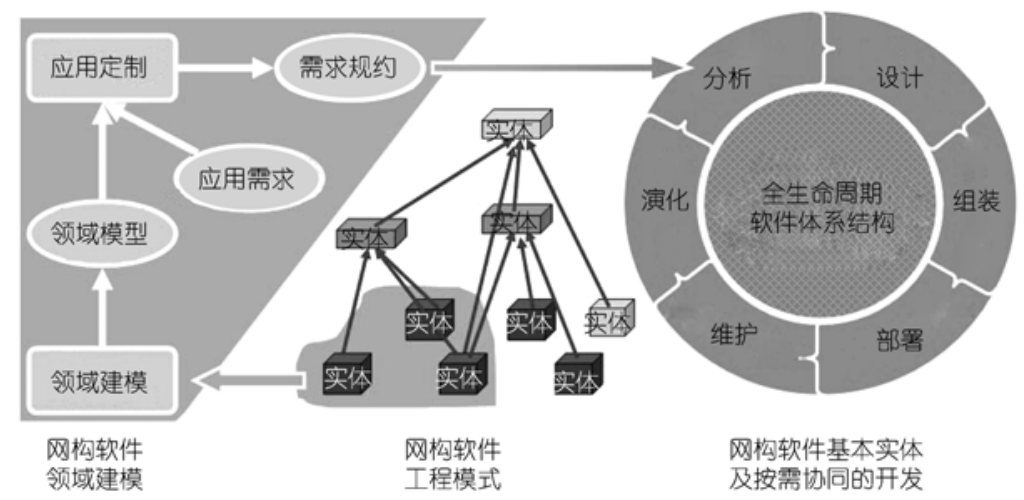

图3 网构软件开发方法体系

调整. 其三，网构软件既服务于处在不同时区的用户也服务于随时到达的其他网构软件的请 求, 而且, 一个网构软件系统本身往往由散布在 Internet 上的其他软件组成, 因此, 一个网构软 件系统一旦交付使用, 往往就不能完全停机. 这意味着改错、优化、增加新功能等活动均需在 线执行, 这些活动也需要经历分析、设计、实现、测试、部署, 而传统软件生存周期中的维护 期难以准确刻画这些活动. 为此, 我们提出了全生命周期软件体系结构的概念, 将软件体系结 构引入到网构软件生命周期的各个阶段, 以适应网构软件的生命周期特点:

- 分析阶段(软件体系结构概念视图): 为了平滑需求与设计之间的鸿沟, 应在需求分析 阶段以结构化的方式来组织问题空间和用户需求. 我们对本阶段制品的具体形式没有要求, 只需能够相对方便、自然、直接地转换为软件体系结构即可. 例如, 面向对象分析的结果和问 题域类图, 可视为一种概念性的软件体系结构; 面向特征的需求分析的结果和特征模型, 则可 采用类似于功能分解的方式半自动地生成概念软件体系结构; 某些面向体系结构的需求分析 方法则直接产生概念软件体系结构.

- 设计阶段(软件体系结构设计视图): 设计者通过研究软件系统的需求规约, 制定相应 的全局设计决策, 进一步细化概念软件体系结构中的构件和连接子, 创建必须的构件和连接 子, 建立静态和动态的软件体系结构模型(包括类型图、实例图和过程图等), 维护需求规约与 软件体系结构之间的映射关系. 需要强调的是, 网构软件的基本宗旨是尽可能复用已有实体, 因此，设计者应在本阶段充分考虑可复用的构件和连接子.

- 组装阶段(软件体系结构实现视图): 本阶段等同于传统软件开发的实现阶段. 与传统 软件开发方法按照设计进行编程以实现目标系统不同, 网构软件的基本功能单元为已经存在 且正在运行中的网构软件实体, 因此, 网构软件实现的重心不是编程而是组装, 即, 选取符合 软件体系结构的实体, 并使所有的实体按照软件体系结构的规定交互. 若实体或交互不符合 软件体系结构的要求, 则需要进行适配, 若适配失败, 则需要开发新的实体以实现目标系统.

- 部署阶段(软件体系结构部署视图): 网构软件大多运行于特定的中间件平台, 需经过 部署才能正常运行. 部署所需的相关信息繁杂, 往往需要部署人员手工填写. 其实, 大部分部 署信息在系统设计与实现阶段已经存在, 经过转换或融合可以复用. 另一方面, 新建实体或新 
的协同可能需要改变已有实体之间的组织关系, 这种组织关系的实现也是部署的主要任务之 一. 为此, 我们定义了软件体系结构部署视图, 它包含了大量由设计视图与实现视图派生出来 的部署信息, 支持以直观的图形方式添加少量的信息, 实时显示目标环境的资源与负载情况, 并支持网构软件实体组织关系的在线调整.

- 维护与演化阶段(软件体系结构运行视图): 从某种意义上来说, 网构软件开发方法可 以看成目标系统软件体系结构以不同视图的方式连续、迭代的细化、映射和转换, 在每一次细 化和转换后, 软件体系结构的语法和语义信息变得更加精确与完整. 到了维护与演化阶段, 运 行视图刻画了系统在运行时刻的实际状态, 因而具有系统最精确和完整的信息. 该视图即为 运行时软件体系结构, 支持对软件系统进行在线维护与演化.

\section{2 网构软件基本实体与协同的开发}

网构软件开发的两个主要任务就是基本实体的开发和按需协同的开发. 具体而言, 网构 软件基本实体本质上是运行于传统单机或局域网的软件系统, 与传统软件的主要区别在于网 构软件自主性、演化性、协同性、多态性、反应性等主要特征. 因此，网构软件基本实体的开 发就是如何开发出具有网构软件特征的传统软件, 以及如何使已有的传统软件演化出网构软 件特征. 为此, 我们提出了基于体系结构、面向构件的软件开发方法ABC(architecture-based component composition) $)^{[19]}$.

以自适应性为例, 对于那些特定于具体应用的自适应, ABC 方法利用软件体系结构的质 量分析和设计来发现需要处理的变化, 基于动态软件体系结构设计和分析确定系统在运行时 刻可以进行的调整, 通过运行时软件体系结构来实施对于体系结构的适应性修改; 对于那些 较为通用的自适应, ABC 方法允许领域专家以不良体系结构模式的形式定义出可能导致可信 问题的不良结构, 并以良好模式给出相应的良好结构, 运行时软件体系结构可自动检测出当 前系统是否具有不良结构, 并自动将其重构为良好结构; ABC 方法还允许在软件体系结构中 加入各种规则, 被赋予规则的实体在运行时通过容器与规则推理引擎绑定, 从而实现基于规 则推理的自适应.

网构软件按需协同处于运行时体系结构的管控之下, 换言之, 按需协同的开发本质上就 是开发出一个管控 Internet 环境下多个基本实体的软件体系结构. 从这个角度看, 以体系结构 为中心的 $\mathrm{ABC}$ 方法仍可用于协同的开发. 但是, 基本实体开发处于一个相对封闭、稳定、可 控的传统运行环境, 而按需协同开发则处于一个开放、动态、难控的 Internet 环境. 因此, 与 采用 $\mathrm{ABC}$ 方法开发网构软件基本实体不同, 按需协同的开发还应考虑构件的分布、自治、异 构等特性，构件交互的多样、复杂、可变等特性.

\section{3 网构软件领域建模}

传统软件系统的开发因所基于的平台相对封闭、静态、稳定, 基本采用自顶向下的途径, 确定系统的范围(即 Scoping)总是建立需求的第一步, 然后通过分解而实施分而治之的策略, 整个开发过程处于有序控制之下. 而网构软件系统的开发所基于的平台是一个软件实体极大 丰富但同时又是开放、动态、难控的框架, 开发活动呈现为通过将原本“无序”的软件实体组合 
为 “有序” 的新实体, 随着时间推移, 这些实体在功能、质量、数量上的变化导致它们再次呈现 出 “无序” 的状态，这种由 “无序”到“有序”的过程往复循环，基本上是一种自底向上、由内向外 的螺旋方式. 只有整合这些无序的实体使之在开发的过程中处于 “有序” 的控制之下, 才能真 正实施前面介绍的基本实体和按需协同的开发.

作为系统化生产可复用软件资产的领域工程, 是在对一类具有相似需求的若干领域成员 系统进行分析的基础上, 考虑领域预期的需求变化、技术演化、经济利益、限制条件等因素, 进 而确定领域的范围, 识别领域中的共性和变化性, 获取一组具有足够可复用性的领域需求, 并 对其进行抽象形成领域模型. 在某种意义上, 领域工程的分析阶段就是一个自底向上、对各个 成员系统进行分析、抽象、构建领域需求模型的过程. 因此, 我们采用领域工程方法和手段对 网构软件实体进行有序化整合: 首先, 通过领域边界的确定和领域分析活动, 把分散在 Internet 各处的无序实体, 组织成具有变化性表示机制的领域模型, 体现一类网构软件系统的 高层目标; 然后, 根据具体的需求经过需求分析对领域模型加以剪裁和扩充, 定制生成包含可 复用基本实体群的需求规约; 最后经开发方法产生新的网构实体或协同. 当网构实体再次变 得“无序”, 又可经过新一轮的领域分析迭代加入领域模型, 从而形成网构软件从“无序”到“有 序”的往复循环过程.

\section{4 结论}

Internet 的发展为信息技术的应用开启了新的篇章, 由此产生了从不同角度或层次系统化 地研究 Internet 技术与应用的多种新型模式或理念, 如, 网格计算、普适计算、服务计算、语 义网、Web Science 等. 基于类似的出发点, 网构软件从软件形态的角度考察开放、动态、难 控的 Internet 环境对软件理论、方法和技术的挑战. 本文总结了近 5 年来我国学者在国家重点 基础研究发展规划支持下的研究与实践成果, 这些成果初步形成了一套以软件体系结构为中 心的网构软件技术体系, 主要包括一种基本实体主体化和按需协同结构化的网构软件模型, 一个实现网构软件模型的自治式网构软件中间件, 以及一种以全生命周期体系结构为中心的 网构软件开发方法.

进一步的工作主要是加强现有成果的深度和广度. 在深度方面, 完善以软件体系结构为 中心的网构软件技术体系, 重点突破网构软件智能可信模型、网构中间件自治管理技术、以及 网构软件开发方法的自动化程度. 在广度方面, 多网融合的大趋势使得软件将运行在一个包 含 Internet、无线网、电信网等多种异构网络的复杂网络环境, 网构软件是否需要以及能否从 Internet 延伸到这种复杂网络环境, 成为我们下一步的主要目标.

\section{参考文献}

1 杨芙清, 梅宏, 吕建, 等. 浅论软件技术发展。电子学报, 2003, 26(9): 1104-1115

2 吕建, 马晓星, 陶先平, 等. 网构软件的研究与进展. 中国科学, E 辑: 信息科学, 2006, 36(10): 1037-1080

3 吕建, 陶先平, 马晓星, 等. 基于 Agent 的网构软件模型研究. 中国科学, E 辑: 信息科学, 2005, 35(12): $1233-1253$

4 Jiao W P, Mei H. Automated adaptations to dynamic software architectures by using autonomous agents. Eng 
Appl Art Intell, 2004(17): 749-770 [DOI]

5 Cao J N, Feng X Y, Lu J, et al. Reliable message delivery for mobile agents: push or pull? IEEE Trans Syst Man Cy A, 2004, 34(5): 577-587 [DOI]

6 Wu W G, Cao J N, Yang J, et al. Design and performance evaluation of efficient consensus protocols for mobile ad hoc networks. IEEE Trans Comput, 2007, 56(08): 1055-1070 [DOI]

7 Cao J N, Ma X X, Chan T S, et al. Architecting and implementing distributed web applications using the graph-oriented approach. Software Pract Exper, 2003, 33: 799-820 [DOI]

8 Liu J, He J F, Liu Z M. A strategy for service realization in service-oriented design. Sci China Ser F-Inf Sci, 2006, 49(6): $864-884$

9 Jiao W P, Mei H. Supporting high interoperability of components by adopting an agent-based approach. Softw Qual J, 2007, 15(3): 283-307 [DOI]

10 黄罡, 王千祥, 曹东刚, 等. 一种面向领域的构件运行支撑平台. 电子学报, 2002, 30(12Z): 39-43

11 Huang G, Mei H, Yang F Q. Runtime software architecture based on reflective middleware. Sci China Ser F-Inf Sci, 2004, 47(5): 555-576 [DOI]

12 黄涛,陈宁江,魏峻,等. OnceAS/Q: 一个面向 QoS 的 Web 应用服务器. 软件学报, 2004, 15(12): 1787-1799

13 Huang G, Liu T C, Mei H, et al. Towards autonomic computing middleware via reflection. In: Proceedings of the 28th Annual International Computer Software and Applications Conference (COMPSAC). Hongkong: IEEE Press, 2004. 122-127

14 Shen J R, Sun X, Huang G, et al. Towards a unified formal model for supporting mechanisms of dynamic component update. In: The 5th Joint Meeting of the European Software Engineering Conference (ESEC-FSE'05) and ACM SIGSOFT Symposium on the Foundations of Software Engineering, Lisbon. New York: ACM, 2005. 80-89

15 梅宏, 曹东刚. ABC-S2C: 一种面向贯穿特性的构件化软件关注点分离技术. 计算机学报, 2005, 28(12): $2036-2044$

16 Wang Q X, Shen J R, Wang X P, et al. A component-based approach to online software evolution. J Softw Maint Evol R, 2006, 18(3): 181-205 [DOI]

17 Huang T, Ding X N, Wei J. An application-semantics-based relaxed transaction model for internetware. Sci China Ser F-Inf Sci, 2006, 49(6): 774-791 [DOI]

18 Huang G, Liu X Z, Mei H. Online approach to feature interaction problems in middleware based system. Sci China Ser F-Inf Sci, 2008, 51(3): 225-239

19 Mei H, Chang J C, Yang F Q. Software component composition based on ADL and middleware. Sci China Ser F-Inf Sci, 2001, 44(2): 136-151

20 张伟, 梅宏. 一种面向特征的领域模型及其建模过程. 软件学报, 14(8): 1345-1356

21 Jin Z, Lu R Q, Bell D A. Automatically multi-paradigm requirements modeling and analyzing: an ontology-based approach. Sci China Ser F-Inf Sci, 2003, 46(4): 279-297

22 Pan Y, Wang L, Zhang L, et al. Relevancy based semantic interoperation of reuse repositories. In: Proceedings of the 12th ACM SIGSOFT Symposium on Foundations of Software Engineering (FSE2004), 2004. 211-220

23 Mei H, Zhang W, Zhao H. A metamodel for modeling system features and their refinement, constraint and interaction relationships. Software Syst Mod, 2006, 5(2): 172-186 [DOI]

24 Hou L S, Jin Z, Wu B D. Modeling and verifying web services driven by requirements: An ontology-based approach. Sci China Ser F-Inf Sci, 2006, 49(6): 792-820 [DOI]

25 Zhao W, Zhang L, Liu Y, et al. SNIAFL: towards a static non-interactive approach to feature location. ACM Trans Softw Eng Meth, 2006, 15(2): 195-226 [DOI]

26 Jiang S J, Xu B W, Shi L. An approach to analyzing recursive programs with exception handling. SIGPLAN Notices, 2006, 4: 30-35 [DOI]

27 谭国真, 李程旭, 刘浩, 等. 交通网格的研究与应用。计算机研究与发展, 2004, 41(12): 2066-2072

28 Mei H, Huang G, Zhao H Y, et al. An architecture centric engineering approach to Internetware. Sci China Ser F-Inf Sci, 2006, 49(6): 702-730 [DOI] 